\begin{tabular}{|c|c|c|c|}
\hline Moosvi, MD & Alexander Abadir, MD & Jacqueline Duong, BS & dyen, MD \\
\hline $\begin{array}{l}\text { Department of Internal Medicine, } \\
\text { Jniversity of California Irvine, } \\
\text { Drange, CA }\end{array}$ & $\begin{array}{l}\text { Department of Internal Medicine, } \\
\text { University of California Irvine, } \\
\text { Orange, CA }\end{array}$ & f California, Irvine & $\begin{array}{l}\text { Department of Gastroenterology and } \\
\text { Hepatology, University of California } \\
\text { Irvine, Orange, CA }\end{array}$ \\
\hline
\end{tabular}

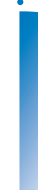

\section{Q: Is it safe to continue biologic agents during surgery in patients with inflammatory bowel disease?}

\begin{abstract}
- Patients with inflammatory bowel 1. disease who are taking monoclonal antibodies against tumor necrosis factor alpha (TNF), interleukins 12 and 23, or integrin can continue taking them around the time of surgery, but small-molecule drugs such as tofacitinib should be withheld.

Inflammatory bowel disease encompasses Crohn disease and ulcerative colitis, and biologic drugs now play an integral role in the treatment of both. Biologic therapy is supplanting thiopurines as the main treatment for inflammatory bowel disease, leading to debate regarding how these drugs should be managed preoperatively in patients undergoing surgery, due to concern for increased risk of postoperative complications.
\end{abstract}

\section{BIOLOGIC AGENTS}

\section{Anti-TNF drugs}

The most studied of the biologics with respect to perioperative management have been the anti-TNF drugs, namely:

- Infliximab and biosimilars such as infliximabs axxq, dyyb, abda, and qbtx

- Adalimumab and biosimilars such as adalimumabs bwwd, afzb, adaz, adbm, bwwd, and atto

- Certolizumab.

\section{Studies of anti-TNF drugs}

While several meta-analyses have attempted to clarify the effect of these drugs on postoperative outcomes, the results have been conflicting.

Dr. Nguyen has disclosed teaching and speaking for Abbvie Pharmaceuticals and Janssen Pharmaceuticals.

doi:10.3949/ccjm.87a.19071
Billioud et $\mathrm{al}^{1}{ }^{1}$ in a meta-analysis, concluded that preoperative anti-TNF use slightly increases overall postoperative complications in patients with inflammatory bowel disease, and particularly infectious complications in patients with Crohn disease.

Ali et $\mathrm{al}^{2}$ in another meta-anlysis, also found a higher risk of complications in patients with Crohn disease receiving preoperative anti-TNF agents (and corticosteroids).

Narula et $\mathrm{al}^{3}$ and Yang et $\mathrm{al}^{4}$ performed meta-analyses demonstrating similar results, both reporting higher rates of total, infectious, and noninfectious postoperative complications in patients with Crohn disease who received infliximab within 30 days before surgery.

Although these studies suggest that antiTNF therapy should be discontinued before surgery, other studies did not detect a higher risk of infectious complications with antiTNF therapy.

Xu et al, ${ }^{5}$ in a 2019 meta-analysis, found no significant difference in the rates of overall, major, minor, infectious, noninfectious, surgical, and medical complications between 1,407 patients with Crohn disease treated preoperatively with infliximab and 4,589 patients who were not.

Yang et $\mathbf{a l}^{6}$ analyzed 13 studies with a total of 2,933 patients with ulcerative colitis receiving infliximab. They similarly found no correlation between infliximab therapy and postoperative morbidity.

Rosenfeld et $\mathbf{a l}^{7}$ reported results similar to those of Yang et al, but in patients with Crohn disease.

Most of the studies included in these metaanalyses were retrospective, were performed at single centers, and had significant heteroge-
Monocloncal antibodies can be continued, but smallmolecule drugs should be withheld 
neity and small sample sizes. They also varied significantly in confounding variables that were not controlled for such as concomitant medical therapy. Steroid use, in particular, is known to increase the risk of infectious complications in patients with ulcerative colitis or Crohn disease in the perioperative period. 8,9

The Postoperative Infection in Inflammatory Bowel Disease (PUCCINI) trial ${ }^{10}$ was perhaps the best study to date to examine postoperative infectious complications in patients with Crohn disease or ulcerative colitis who had been treated preoperatively with anti-TNF drugs. It prospectively enrolled 955 patients undergoing abdominal surgery, of whom 382 had been treated with anti-TNFs preoperatively and 573 had not. Serum antiTNF levels were measured preoperatively and reported as either detectable or undetectable.

The investigators found no significant increases in any infection $(19.4 \%$ vs $20.2 \%, P$ $=.80)$ or surgical site infections $(12.3 \%$ vs $12.7 \%, P=.92)$ in the anti-TNF recipients compared with nonrecipients. Further, detectable serum anti-TNF levels were not associated with higher rates of any infection. ${ }^{10}$

As the first large prospective study of its

The PUCCINI trial is currently the best source of information

\section{on preoperative}

management

of anti-TNF

therapy kind, the PUCCINI trial is currently the best source of information on the preoperative management of anti-TNF therapy in inflammatory bowel disease patients undergoing abdominal surgery.

\section{Ustekinumab}

Ustekinumab, a monoclonal antibody targeting interleukins 12 and 23, was approved in 2016 for use in Crohn disease, though it was previously used off-label for patients for whom anti-TNF therapy failed. After induction, ustekinumab maintenance therapy is given every 8 weeks.

In a multicenter Canadian study ${ }^{11}$ comparing preoperative anti-TNF use and preoperative ustekinumab use in patients with Crohn disease undergoing abdominal surgery, there were no significant differences in postoperative complications, length of hospital stay, or mortality rates.

These results were mirrored in a US study of 44 ustekinumab-treated patients and 169 anti-TNF-treated patients undergoing major abdominal surgery. There was no difference in postoperative surgical site infection rates or hospital readmission rates between the 2 cohorts. $^{12}$

\section{Anti-integrin antibodies}

Vedolizumab and natalizumab are the most widely used anti-integrin antibodies. The former is a gut-specific antibody that selectively inhibits lymphocyte trafficking and inflammatory response in the gastrointestinal tract. It is given every 8 weeks after induction but can be dosed as often as every 4 weeks in patients with severe disease or those who lose response to initial treatment. ${ }^{13}$

\section{Studies of anti-integrin antibodies}

Law et al $^{14}$ conducted a meta-analysis comparing 5 studies with 307 patients receiving vedolizumab, 490 patients receiving anti-TNF drugs, and 535 patients not receiving biologic agents. The analysis revealed no significant difference in rates of overall postoperative complications or infectious complications between those on vedolizumab and either those on anti-TNF agents or those with no biologic exposure.

Yung et $\mathbf{a l}^{15}$ recently performed another meta-analysis of 4 studies in 1,080 patients with Crohn disease or ulcerative colitis, who had vedolizumab exposure, anti-TNF exposure, or no exposure to biologics. The ulcerative colitis patients with vedolizumab exposure did have a significantly lower overall postoperative complication rate compared with those with anti-TNF exposure. However, there were no other significant differences in infectious, surgical site, or major complications in those with either ulcerative colitis or Crohn disease between vedolizumab and anti-TNF or vedolizumab and no biologics. Likewise, there were no significant differences in the rates of additional surgery for complications. ${ }^{15}$

\section{SMALL-MOLECULE DRUGS}

\section{Tofacitinib, a JAK inhibitor}

Tofacitinib, approved in May 2018, is one of the newest medications for moderate to severe ulcerative colitis. This small molecule is a Janus kinase (JAK) inhibitor that is taken orally twice a day. It has been approved for use in rheumatoid arthritis since $2012 .{ }^{16}$ 
As tofacitinib is a recent addition to inflammatory bowel disease therapy, there are limited data assessing its perioperative use. Current guidelines from the American College of Rheumatology (ACR) and the American Association of Hip and Knee Surgeons (AAHKS) recommend holding tofacitinib starting 7 days before total knee or total hip surgery due to increased risk of infectious complications. It can be resumed as early as 14 days after surgery. ${ }^{17}$

\section{OUR RECOMMENDATIONS}

\section{Biologics}

In summary, to date, several single-center retrospective reviews, prospective studies, and meta-analyses exploring postoperative complications in inflammatory bowel disease patients on anti-TNF therapy have demonstrated mixed results. However, nearly all of the earlier studies had notable weaknesses, including marked heterogeneity among studies in meta-analyses and lack of controlled variables. On the contrary, the landmark PUCCINI trial has emerged as the most instrumental study of preoperative anti-TNF therapy in patients with inflammatory bowel disease, owing to its robust study design and high power. Given the compelling results of the PUCCINI trial, in addition to several negative studies examining vedolizumab and ustekinumab, we recommend that all biologic therapy be continued preoperatively in both Crohn disease and ulcerative colitis patients.

However, biologic therapy does not need to be continued preoperatively in all cases. For instance, in patients with stricturing Crohn disease undergoing intestinal resection with no plans for biologic therapy postoperatively, preoperative biologic therapy may not be warranted.

As demonstrated by the PUCCINI trial, there is no benefit in measuring serum antiTNF levels preoperatively. Further, drug levels

\section{REFERENCES}

1. Billioud V, Ford AC, Tedesco ED, Colombel JF, Roblin X, PeyrinBiroulet L. Preoperative use of anti-TNF therapy and postoperative complications in inflammatory bowel diseases: a meta-analysis. J Crohns Colitis 2013; 7(11):853-867. doi:10.1016/j.crohns.2013.01.014

2. Ali UA, Martin ST, Rao AD, Kiran RP. Impact of preoperative immunosuppressive agents on postoperative outcomes in Crohn's disease. Dis Colon Rectum 2014; 57(5):663-674. doi:10.1097/DCR.0000000000000099 of other biologic agents have yet to be studied, and thus in the absence of any supporting data, its practice should not be adopted.

The above recommendations pertain to intra-abdominal surgeries only, as only these surgeries were included in the referenced studies.

\section{Small molecules}

With respect to tofacitinib, in the absence of any data examining its perioperative use in inflammatory bowel disease patients, our recommendation reflects the 2017 ACR/AAHKS guidelines based on evidence from prior studies in patients with rheumatic diseases. These guidelines reflect the markedly short half-life of tofacitinib, which is 3.2 hours. ${ }^{16}$ Therefore, we recommend holding the medication 7 days before surgery and resuming as early as 14 days after surgery. It should be noted, however, that for urgent or emergent situations, we would not delay surgery due to any biologic or small molecule therapy. The increased risk of postoperative complications, if even present, does not outweigh the risks of delaying surgery.

Selective JAK inhibitors, namely filgotinib, upadacitinib, and peficitinib, have shown promising preliminary results in clinical trials and may soon be approved for treating inflammatory bowel disease. Because these drugs are so new, their influence on operative outcomes at this time is unknown.

Our recommendations differ from those included in the 2017 professional society guidelines, ${ }^{17}$ which recommend holding all biologic medications as close to 1 dosing cycle as possible before all elective procedures. The deviation of our recommendations from these guidelines is due to the recent emergence of groundbreaking studies such as the PUCCINI trial which have allayed fears of increased risks of postoperative complications with biologic use in inflammatory bowel disease patients.

\section{Biologic therapy does not need to be continued preoperatively in all cases}

3. Narula N, Charleton D, Marshall JK. Meta-analysis: peri-operative anti-TNFa treatment and post-operative complications in patients with inflammatory bowel disease. Aliment Pharmacol Ther 2013; 37(11):1057-1064. doi:10.1111/apt.12313

4. Yang ZP, Hong L, Wu Q, Wu KC, Fan DM. Preoperative infliximab use and postoperative complications in Crohn's disease: a systematic review and meta-analysis. Int J Surg 2014; 12(3):224-230. doi:10.1016/j.ijsu.2013.12.015

5. Xu YY, Yang LS, An P, Zhou B, Liu G. Meta-analysis: the influ- 
ence of preoperative infliximab use on postoperative complications of Crohn's disease. Inflamm Bowel Dis 2019; 25(2):261-269. doi:10.1093/ibd/izy246

6. Yang Z, Wu Q, Wang F, Wu K, Fan D. Meta-analysis: effect of preoperative infliximab use on early postoperative complications in patients with ulcerative colitis undergoing abdominal surgery. Aliment Pharmacol Ther 2012; 36(10):922-928. doi:10.1111/apt.12060

7. Rosenfeld G, Qian H, Bressler B. The risks of post-operative complications following pre-operative infliximab therapy for Crohn's disease in patients undergoing abdominal surgery: a systematic review and meta-analysis. J Crohns Colitis 2013; 7(11):868-877. doi:10.1016/j.crohns.2013.01.019

8. Zangenberg MS, Horesh N, Kopylov U, El-Hussuna A. Preoperative optimization of patients with inflammatory bowel disease undergoing gastrointestinal surgery: a systematic review. Int J Colorectal Dis 2017; 32(12):1663-1676. doi:10.1007/s00384-017-2915-4

9. Ferrante M, D'Hoore A, Vermeire S, et al. Corticosteroids but not infliximab increase short-term postoperative infectious complications in patients with ulcerative colitis. Inflamm Bowel Dis 2009; 15(7):1062-1070. doi:10.1002/ibd.20863

10. Cohen BL, Fleshner P, Kane SV, et al. 415a-Anti-tumor necrosis factor therapy is not associated with post-operative infection: results from prospective cohort of ulcerative colitis and Crohn's disease patients undergoing surgery to identify risk factors for postoperative infection I (PUCCINI) [abstract]. Gastroenterology 2019; 156(6 suppl 1):S-80. doi:10.1016/S0016-5085(19)36987-2

11. Shim HH, Ma C, Kotze PG, et al. Preoperative ustekinumab treatment is not associated with increased postoperative complications in Crohn's disease: a Canadian multi-centre observational cohort study. J Can Assoc Gastroenterol 2018; 1(3):115-123. doi:10.1093/jcag/gwy013

12. Lightner AL, McKenna NP, Tse CS, et al. Postoperative outcomes in ustekinumab-treated patients undergoing abdominal operations for Crohn's disease. J Crohns Colitis 2018; 12(4):402-407. doi:10.1093/ecco-jcc/jjx163

13. Lightner AL. Perioperative management of biologic and immunosuppressive medications in patients with Crohn's disease. Dis Colon Rectum 2018; 61(4):428-431. doi:10.1097/DCR.0000000000001072

14. Law CC, Narula A, Lightner AL, McKenna NP, Colombel JF, Narula N. Systematic review and meta-analysis: preoperative vedolizumab treatment and postoperative complications in patients with inflammatory bowel disease. J Crohns Colitis 2018; 12(5):538-545. doi:10.1093/ecco-jcc/jjy022

15. Yung DE, Horesh N, Lightner AL, et al. Systematic review and metaanalysis: vedolizumab and postoperative complications in inflammatory bowel disease. Inflamm Bowel Dis 2018; 24(11):2327-2338. doi:10.1093/ibd/izy156

16. Rosenberg J, Steinberg JM, Mattar MC. Tofacitinib for the treatment of ulcerative colitis: a review of the literature. World J MetaAnal 2019; 7(8):373-379. doi:10.13105/wjma.v7.i8.373

17. Goodman SM, Springer B, Guyatt G, et al. 2017 American College of Rheumatology/American Association of Hip and Knee Surgeons Guideline for the perioperative management of antirheumatic medication in patients with rheumatic diseases undergoing elective total hip or total knee arthroplasty. J Arthroplasty 2017; 32(9):26282638. doi:10.1016/j.arth.2017.05.001

Address: Zain Moosvi, MD, Department of Internal Medicine, University of California Irvine, 101 The City Drive S, Orange, CA 92868; zmoosvi@uci.edu 